\title{
BMJ Open Simple scoring algorithm to identify community-dwelling older adults with limited health literacy: a cross-sectional study in Taiwan
}

\author{
Wen-Hsuan Hou (D , 1,2,3,4 Ken N Kuo, ${ }^{3,5}$ Mu-Jean Chen, ${ }^{6}$ Yao-Mao Chang, ${ }^{7,8}$ \\ Han-Wei Tsai, ${ }^{1}$ Ding-Cheng Chan, ${ }^{9,10}$ Chien-Tien Su, ${ }^{11,12,13}$ Der-Sheng Han, ${ }^{14,15}$ \\ Hsiu-Nien Shen, ${ }^{16}$ Chung-Yi Li (D) ${ }^{17,18,19}$
}

To cite: Hou W-H, Kuo KN, Chen M-J, et al. Simple scoring algorithm to identify community-dwelling older adults with limited health literacy: a cross-sectional study in Taiwan. BMJ Open 2021;11:e045411. doi:10.1136/ bmjopen-2020-045411

- Prepublication history and additional supplemental material for this paper are available online. To view these files, please visit the journal online (http://dx.doi.org/10.1136/ bmjopen-2020-045411).

H-NS and C-YL contributed equally.

Received 30 September 2020 Accepted 15 0ctober 2021

Check for updates

(c) Author(s) (or their employer(s)) 2021. Re-use permitted under CC BY-NC. No commercial re-use. See rights and permissions. Published by BMJ.

For numbered affiliations see end of article.

Correspondence to

Dr Chung-Yi Li;

cyli99@mail.ncku.edu.tw

\section{ABSTRACT}

Objective Health literacy $(\mathrm{HL})$ is the degree of individuals' capacity to access, understand, appraise and apply health information and services required to make appropriate health decisions. This study aimed to establish a predictive algorithm for identifying community-dwelling older adults with a high risk of limited HL.

Design A cross-sectional study.

Setting Four communities in northern, central and southern Taiwan.

Participants A total of 648 older adults were included. Moreover, $85 \%$ of the core data set was used to generate the prediction model for the scoring algorithm, and 15\% was used to test the fitness of the model.

Primary and secondary outcome measures Pearson's $\chi^{2}$ test and multiple logistic regression were used to identify the significant factors associated with the HL level. An optimal cut-off point for the scoring algorithm was identified on the basis of the maximum sensitivity and specificity.

Results A total of 350 (54.6\%) patients were classified as having limited $\mathrm{HL}$. We identified 24 variables that could significantly differentiate between sufficient and limited HL. Eight factors that could significantly predict limited HL were identified as follows: a socioenvironmental determinant (ie, dominant spoken dialect), a health service use factor (ie, having family doctors), a health cost factor (ie, self-paid vaccination), a heath behaviour factor (ie, searching online health information), two health outcomes (ie, difficulty in performing activities of daily living and requiring assistance while visiting doctors), a participation factor (ie, attending health classes) and an empowerment factor (ie, self-management during illness). The scoring algorithm yielded an area under the curve of 0.71 , and an optimal cut-off value of 5 represented moderate sensitivity (62.0\%) and satisfactory specificity (76.2\%).

Conclusion This simple scoring algorithm can efficiently and effectively identify community-dwelling older adults with a high risk of limited HL.

\section{INTRODUCTION}

Health literacy (HL), which refers to people's knowledge and competency required to meet the complex demands of health and
Strengths and limitations of this study

- Our health literacy $(\mathrm{HL})$ prediction model comprising personal, contextual, and socioenvironmental factors can be used to formulate health policies for older adults.

- This scoring algorithm not only helps clinicians assess and identify the HL level among older adults but also assists researchers to establish early identification for older adults with limited HL.

- Prospective trials should examine the implementation and utility of this simple scoring algorithm for the early detection of older adults with a high risk of limited HL in the community.

healthcare (HC), is crucial and increasingly being recognised in modern society. However, no consensus exists regarding the definition, dimensions and conceptual models of HL. The European Health Literacy Consortium has proposed a conceptual model integrating medical and public health perspectives of HL; the model can be used to determine the different dimensions of HL across different clinical and community settings. They defined HL as the ability to access, understand, appraise and apply health information or services for obtaining appropriate $\mathrm{HC}$ and for disease prevention (DP) and health promotion (HP). ${ }^{1}$ The HL measure has been indicated as the sixth vital sign along with temperature, pulse, respiration, blood pressure and pain level. The importance of HL should be emphasised, so that clinicians and public health workers can enable early and precise access to intervention strategies. ${ }^{23}$ However, the construct of HL is complex and dynamic and encompasses many aspects of individuals' use of health information and the HC system. Therefore, the European Health Literacy Survey (HLS-EU) Consortium has 
proposed a theoretical model that integrates medical and public health perspectives of HL and that accounts for its various antecedents including personal, contextual, social and environmental determinants. Therefore, the level of HL indicates health service use, health costs, health behaviour, health outcomes, participation and empowerment among individuals. ${ }^{1}$

Older adults generally have more chronic illnesses and less formal education than their younger counterparts. ${ }^{4}$ Moreover, older adults experience unique problems related to physical and cognitive functioning that cause difficulty in finding accurate health information and using appropriate HC services. ${ }^{5}$ Several national surveys have reported that more than half of older adults have limited HL. ${ }^{6-8}$ Studies have reported that the level of HL was significantly lower in older people than in younger people. ${ }^{9-11}$ Moreover, limited HL results in poor health outcomes $^{12}$ and health behaviours, ${ }^{13}$ increased HC expenditure $^{14}$ and health service usage ${ }^{15}$ and inadequate empowerment ${ }^{16}$ and participation. ${ }^{17}$ Therefore, early and accurate prediction of limited HL among older adults is essential to implement prompt and appropriate $\mathrm{HC}$ strategies.

Various HL measures have been developed for older adults. ${ }^{18}$ A US national survey examined the agreement (sensitivity and specificity) and discrimination ( $c$-statistic) of functional HL derived from common sociodemographic data; this functional HL can serve as a readily calculated HL proxy score. ${ }^{19}$ A study developed a predictive model by performing multiple regression and validating its efficiency in identifying older people with the highest risk of inadequate functional HL ${ }^{20}$ However, most of these studies lack underlying theoretical basis and fail to sufficiently cover the comprehensive dimensions of HL across different clinical environments. Furthermore, to the best of our knowledge, no study has developed a prediction model of self-reported general HL for the early and accurate identification of HL levels in older adults. Therefore, developing a simple algorithm that can be applied in clinical settings to accurately identify older adults with a high risk of limited HL is essential. This study conducted a survey among community-dwelling older adults to identify factors predicting limited HL and constructed an optimal scoring algorithm for predicting HL.

\section{METHODS}

\section{Patient and public involvement}

Figure 1 presents information on participant selection, data set division and analysis procedures. In this crosssectional study, by adopting the convenience sampling method, we recruited eligible community participants aged $\geq 65$ years from six senior service centres and three health check-up clinics in northern, central and southern Taiwan between June and September 2018. The

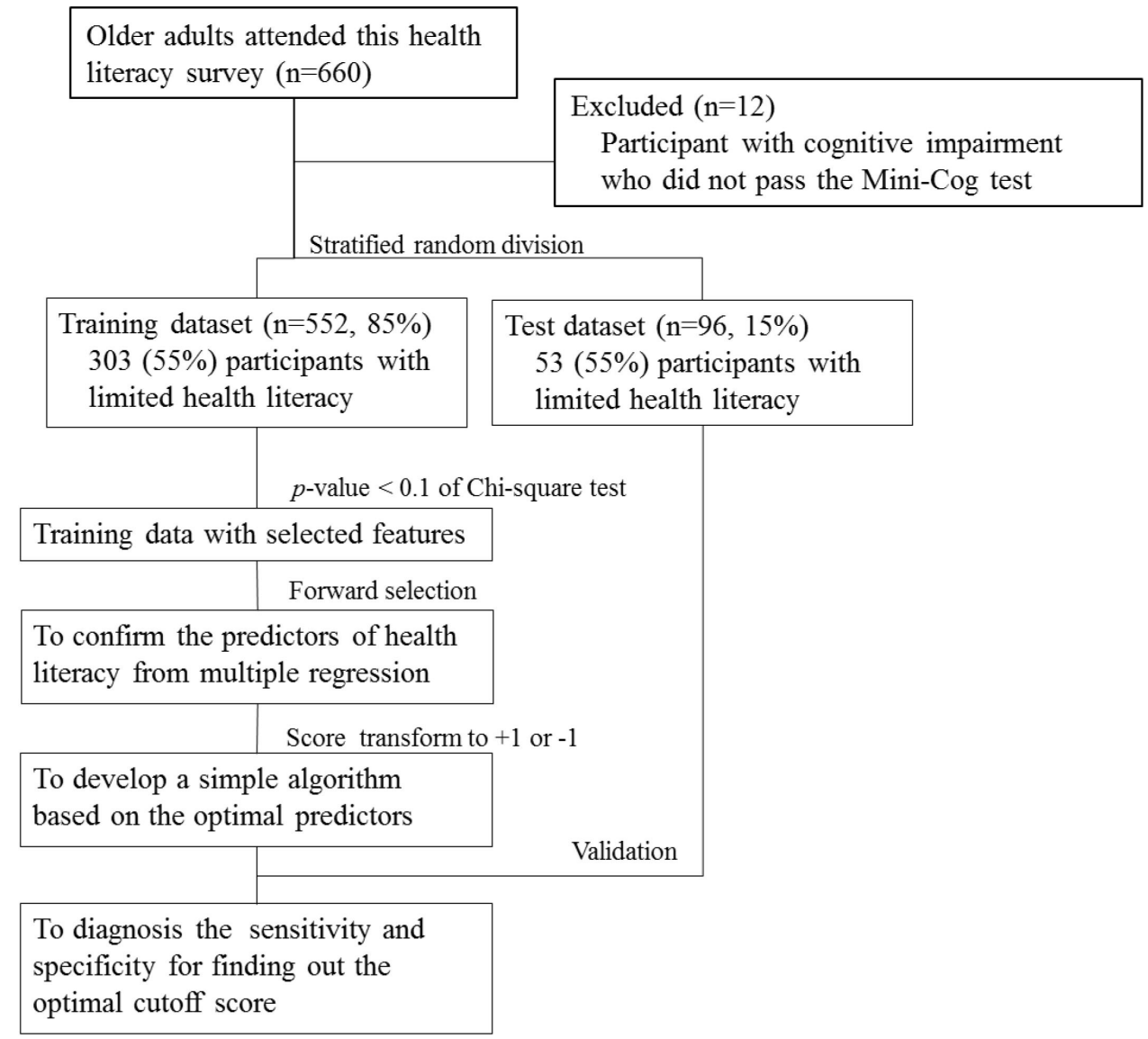

Figure 1 Flow chart of participant selection, data set division and analysis procedure. 
individuals were excluded from the study if they were found to be cognitively impaired based on the Mini-Cog instrument screening. The survey questionnaire items and self-reported outcome measures were developed following the conclusions of three consensus meetings among multidisciplinary experts of health policy, HL, health education, social welfare, gerontology and geriatric medicine. We either interviewed participants to obtain responses for the questionnaire or asked them to complete a self-administered HL-related questionnaire. ${ }^{21}$ Trained interviewers explained the objective of the study to respondents before they expressed their willingness to participate in the study. The survey was anonymous, and respondents were allowed to suspend the interview at any time. There was no difference in expected medical treatment whether the individual join or turn down our study. The participants were allowed to withdraw anytime or complete the interview in different time schedule if they felt tired or stressful. The study results were disseminated to the public through a conference presentation hosted by the Taiwan National Health Research Institutes at the end of the study. The minimum sample size was decided from the 10:1 ratio of participants to variables by Everitt's recommendations. ${ }^{22}$

\section{Procedure}

After signing informed consent forms for participation in the study, the participants were either interviewed or asked to complete a self-administered questionnaire including questions on 52 potential predictors including personal, situational, and socioenvironmental determinants and factors related to health service use, health costs, health behaviour, health outcomes, participation and empowerment based on the theoretical model of the HLS-EU Consortium. ${ }^{23}$

\section{Outcome measures}

The 47-item HLS-EU Questionnaire (HLS-EU-Q), developed by the HLS-EU Consortium, was used to assess the comprehensive HL of the study participants. The HLS-EU-Q measures four HL competencies (access, understand, appraise and apply health information) required under three health domains: HC (16 items), DP (15 items) and HP (16 items). Each item assesses the self-perceived difficulty in performing selected healthrelated tasks on a 4-point scale ranging from 'very easy' (4) to 'very difficult' (1). Higher scores indicate a higher level of HL. For ease of comparison, the score of each domain (ie, HC, DP and HP) was linearly transformed to a score between 0 and 50 by using a scale adopted in the HLS-EU, which $h$ has been validated to have satisfactory psychometric properties. ${ }^{24}$ On the basis of the scores, HL was divided into four categories: inadequate (0-25), problematic (26-33), sufficient (34-42) and excellent (43-50). ${ }^{25} 26$ We dichotomised HL into 'sufficient' and 'limited' based on a cut-off value of 34 , as defined by the HLS-EU. ${ }^{25}$

\section{Statistical analysis}

The dichotomised outcome is defined using the HL level as follows:

$$
\mathrm{y}=\left\{\begin{array}{c}
1, \quad \text { low HL }[\text { probability }=p] \\
0, \quad \text { high HL }[\text { probability }=1-\mathrm{p}]
\end{array}\right.
$$

To develop a scoring algorithm for predicting a low level of HL, the core data set was divided using stratified random sampling without the replacement method as follows: $85 \%$ of the core data set was categorised into the training data set, which was used for training the prediction model to create the scoring rule, and $15 \%$ of the data set was categorised as the validation data set, which was used for validating the scoring algorithm (figure 1). ${ }^{27} \mathrm{By}$ Everitt's recommendations, a minimum of 520 participants should be included in the training data. ${ }^{22}$ Pearson's $\chi^{2}$ test was used initially to reduce variables and ensure that variables of crucial dimensions were retained. In addition, Pearson's $\chi^{2}$ test was used to evaluate the association of the HL level with each of the 52 predictors included in the self-administered questionnaire. ${ }^{28}$ If the variables were not answered, we would not include the record in the analysis. To select the most relevant predictors, variables with a $\mathrm{p}<0.1$ were included in multiple logistic regressions. Multiple logistic regressions with forward selection were used to examine relationships between limited HL and potential predictors classified into the domains of personal determinants, situational determinants, socioenvironmental determinants, health service use, health costs, health behaviour, health outcomes, participation and empowerment. Furthermore, the potential predictors with a $\mathrm{p}<0.05$ were identified from the multiple logistic regression model. ${ }^{29} 30$ The formula used for multiple regression was as follows:

$$
\begin{aligned}
\operatorname{logit}(p) & =\log \left[\frac{p}{1-p}\right]=\alpha+\beta_{1} x_{1}+\cdots+\beta_{i} x_{i} \\
p & =\frac{\exp ^{\alpha+\beta_{1} x_{1}+\cdots+\beta_{i} x_{i}}}{1+\exp ^{\alpha+\beta_{1} x_{1}+\cdots+\beta_{i} x_{i}}}, 0 \leq p \leq 1
\end{aligned}
$$

where $p$ denotes the probability of limited HL in older adults, $\alpha$ is the intercept of multiple regression, and $\beta$ is the slope of the specified predictor from 1st, $2 \mathrm{nd}, \ldots$, ith. In multiple regression, the OR was estimated using $\exp \left(\beta_{i}\right)$ to present relationships between dichotomised HL and predictors, and the estimated ORs were applied to calculate the clinical score for predicting health risk. ${ }^{31} 32$ To develop a simple algorithm, a previous study developed a new score based on the summation of significant predictors identified from multiple logistic regression. Significant predictors that were positively associated with limited HL $(\mathrm{OR}>1)$ were assigned a value of +1 , whereas those that were negatively associated with limited HL $(\mathrm{OR}<1)$ were assigned a value of $-1 .^{31}$

Separately, $15 \%$ of the participants were included to validate the proposed scoring algorithm. On the basis of the algorithm obtained from the training data set, the total score was calculated for each older adult in the test dataset. The overall accuracy of prediction of limited HL 
using this score was evaluated by calculating sensitivity, specificity, positive predictive value (PPV) and negative predictive value (NPV) ${ }^{33}$ The model fit was assessed on the basis of McFadden's pseudo R-square (measuring the reduction in the maximised log-likelihood from the intercept only model) and $c$-statistic (area under (AUC) the receiver operating characteristic (ROC) curve) values. ${ }^{3435}$ A two-sided 95\% CI for the AUC was used to denote uncertainty, ${ }^{36}$ and a $p>0.05$ in the Hosmer-Lemeshow test was used to indicate algorithm performance. As a graphical illustration of sensitivity-specificity trade-offs at each cutoff, the ROC curve demonstrated the degree to which the HL score could discriminate the level of HL, and the best cut-off value corresponded to a perfect scenario $(100 \%$ sensitivity and $100 \%$ specificity). However, in practice, the optimal cut-off value should maintain a balance between sensitivity and specificity, ${ }^{37}$ which refers to the number of predictors calculated using the optimal classification threshold in our simple scoring algorithm. All statistical analyses were performed using SAS V.9.4 (SAS Institute).

\section{RESULTS}

A total of 648 older adults were recruited. We excluded 12 patients who were found to have mild cognitive impairment by using the Mini-Cog instrument. Figure 1 presents age-specific HL levels. Nearly half $(41.6 \%-46.1 \%)$ of the participants had problematic HL. A large proportion of the participants $(72.6 \%)$ aged $\geq 81$ years had limited HL. Table 1 lists the sociodemographic characteristics of the participants in the training data set $(n=552 / 648)$ and test data set $(n=96 / 648)$. Sex, age, educational level, marital status, occupation and monthly income showed similar distribution between the training and test data sets.

In the training data set $(n=552 / 648)$, of the 52 variables included in the original questionnaire (online supplemental appendix 1), 24 (ie, 5 personal determinants, 2 situational determinants, 2 socioenvironmental determinants, 1 factor related to health service use, 1 factor related to health costs, 3 factors related to health behavior, 6 factors related to health outcomes, 1 factor related to participation, and 3 factors related to empowerment of

Table 1 Sociodemographic characteristics of study participants across the core, training and test data sets

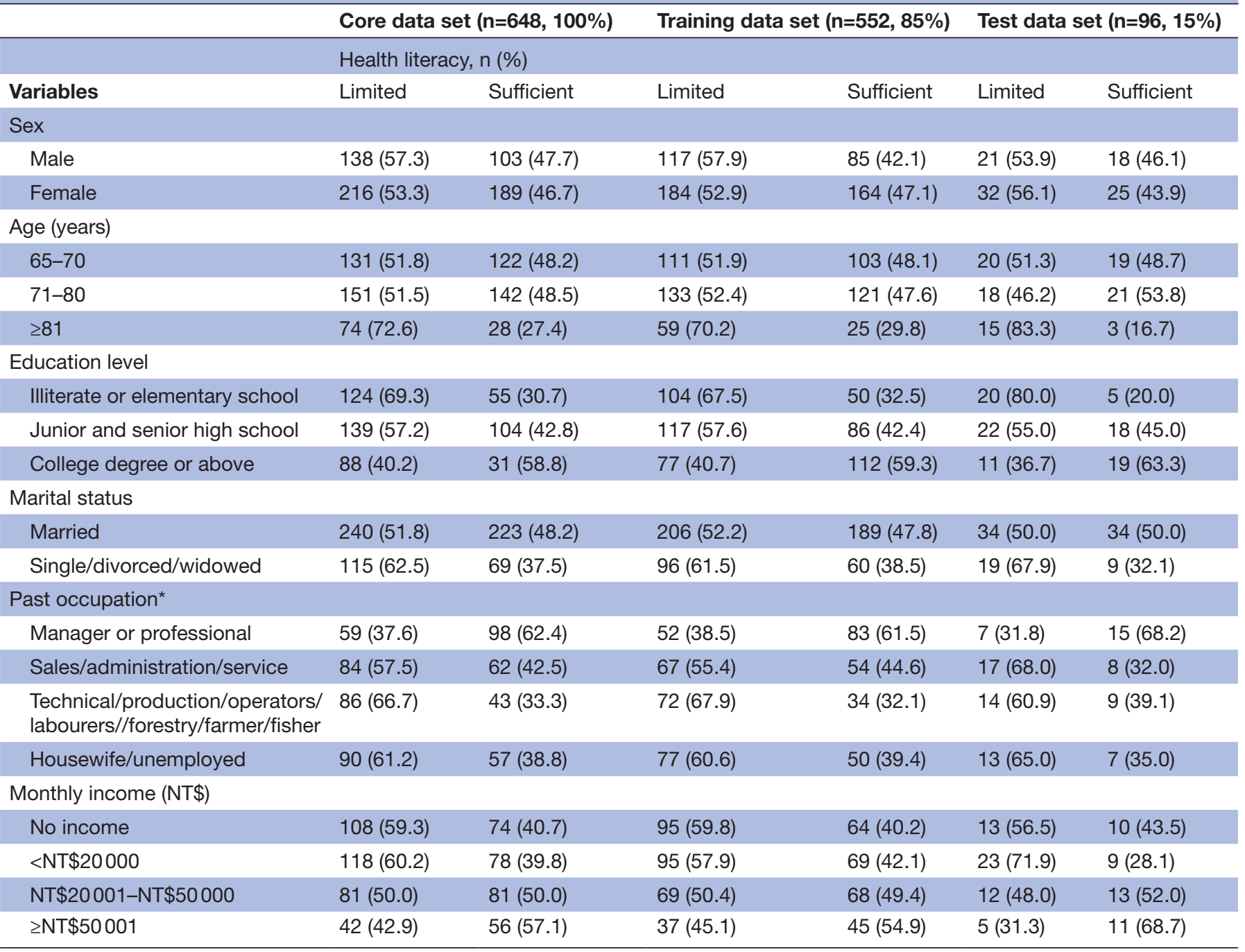

${ }^{*}$ Denoted missing participants $(n=579)$. 
Table 2 The 24 factors significantly correlated with health literacy level based on Pearson's $\chi^{2}$ tests in the training dataset $(n=552,85 \%)$

\begin{tabular}{|c|c|c|c|c|c|c|}
\hline \multirow[b]{2}{*}{ Scope and predictor } & \multicolumn{4}{|c|}{ Health literacy, n (\%) } & \multirow[b]{2}{*}{$x^{2}$} & \multirow[b]{2}{*}{$P$ value } \\
\hline & \multicolumn{2}{|l|}{ Limited } & \multicolumn{2}{|l|}{ Sufficient } & & \\
\hline \multicolumn{7}{|l|}{ Personal determinants } \\
\hline Yes & 12 & $(4.0)$ & 23 & $(9.4)$ & 6.5 & 0.011 \\
\hline No & 287 & $(96.0)$ & 222 & (90.6) & & \\
\hline Junior and senior high school & 117 & (39.3) & 86 & (34.7) & & \\
\hline College degree or above & 77 & $(25.8)$ & 112 & $(45.2)$ & & \\
\hline \multicolumn{7}{|l|}{ Past occupation ( $n=489$ ) } \\
\hline Manager or professional & 52 & $(19.4)$ & 83 & (37.6) & 23.6 & $<0.001$ \\
\hline \multicolumn{7}{|l|}{ Age $(n=552$, years $)$} \\
\hline $65-70$ & 111 & $(36.6)$ & 103 & $(41.4)$ & 9.4 & 0.009 \\
\hline $71-80$ & 133 & $(43.9)$ & 121 & $(48.6)$ & & \\
\hline$\geq 81$ & 59 & $(19.5)$ & 25 & $(10.0)$ & & \\
\hline \multicolumn{7}{|l|}{ Monthly income $(n=542, N T \$)$} \\
\hline No income & 95 & $(32.1)$ & 64 & $(26.0)$ & 6.4 & 0.094 \\
\hline$<\mathrm{NT} \$ 20000$ & 95 & $(32.1)$ & 69 & (28.0) & & \\
\hline NT\$20001-NT\$50000 & 69 & $(23.3)$ & 68 & $(27.6)$ & & \\
\hline$\geq \mathrm{NT} \$ 50001$ & 37 & $(12.5)$ & 45 & $(18.3)$ & & \\
\hline Taiwanese, Hakka, or other dialect & 154 & $(51.0)$ & 41 & $(16.5)$ & 71.2 & $<0.001$ \\
\hline Mandarin & 148 & $(49.0)$ & 208 & (83.5) & & \\
\hline \multicolumn{7}{|l|}{ Residential area $(n=552)$} \\
\hline Taipei city & 158 & $(52.1)$ & 157 & $(63.1)$ & 6.6 & 0.010 \\
\hline Other cities & 145 & $(47.9)$ & 92 & (36.9) & & \\
\hline \multicolumn{7}{|l|}{ Health service use } \\
\hline \multicolumn{7}{|l|}{ Having a family doctor $(n=548)$} \\
\hline Yes & 16 & $(5.3)$ & 24 & $(9.8)$ & 4.0 & 0.046 \\
\hline No & 286 & $(94.7)$ & 222 & $(90.2)$ & & \\
\hline \multicolumn{7}{|l|}{ Health costs } \\
\hline \multicolumn{7}{|l|}{ Pneumonia self-paid vaccination $(n=547)$} \\
\hline Yes & 85 & $(28.4)$ & 100 & $(40.3)$ & 8.6 & 0.003 \\
\hline No & 214 & $(71.6)$ & 148 & $(59.7)$ & & \\
\hline \multicolumn{7}{|l|}{ Health behaviours } \\
\hline Exercise frequency $(n=550)$ & & & & & & \\
\hline No exercise & 63 & $(20.9)$ & 27 & (10.9) & 9.9 & 0.007 \\
\hline Every day & 114 & $(37.7)$ & 107 & $(43.1)$ & & \\
\hline
\end{tabular}


Open access

Table 2 Continued

\begin{tabular}{|c|c|c|c|c|c|c|}
\hline \multirow[b]{2}{*}{ Scope and predictor } & \multicolumn{4}{|c|}{ Health literacy, n (\%) } & \multirow[b]{2}{*}{$x^{2}$} & \multirow[b]{2}{*}{$P$ value } \\
\hline & Limited & & Sufficient & & & \\
\hline Weekly or monthly & 125 & $(41.4)$ & 114 & $(46.0)$ & & \\
\hline No & 63 & $(20.9)$ & 22 & $(8.9)$ & 26.3 & $<0.001$ \\
\hline Sometimes & 172 & $(57.1)$ & 130 & $(52.4)$ & & \\
\hline Yes & 168 & $(56.8)$ & 156 & $(64.2)$ & 3.1 & 0.079 \\
\hline No & 128 & $(43.2)$ & 87 & (35.8) & & \\
\hline \multicolumn{7}{|c|}{ Searching online health information $(n=548)$} \\
\hline Yes & 75 & $(24.9)$ & 107 & $(43.3)$ & 20.7 & $<0.001$ \\
\hline Need assistance & 50 & $(16.6)$ & 11 & $(4.4)$ & 20.4 & $<0.001$ \\
\hline No assistance needed & 251 & $(83.4)$ & 237 & $(95.6)$ & & \\
\hline \multicolumn{7}{|l|}{ Diabetes mellitus $(n=549)$} \\
\hline Yes & 58 & (19.21) & 34 & $(13.77)$ & 2.9 & 0.090 \\
\hline No & 244 & $(80.79)$ & 213 & (86.23) & & \\
\hline \multicolumn{7}{|l|}{ Hypertension $(n=549)$} \\
\hline Yes & 155 & $(51.3)$ & 101 & $(40.9)$ & 5.9 & 0.015 \\
\hline No & 147 & $(48.7)$ & 146 & $(59.1)$ & & \\
\hline \multicolumn{7}{|l|}{ Self-care $(n=550)$} \\
\hline \multicolumn{7}{|c|}{ Activities of daily living $(n=551)$} \\
\hline Yes & 63 & $(20.9)$ & 32 & $(13.0)$ & 5.8 & 0.016 \\
\hline No & 239 & $(79.1)$ & 214 & $(87.0)$ & & \\
\hline \multicolumn{7}{|l|}{ Participation } \\
\hline \multicolumn{7}{|c|}{ Attending health classes $(n=547)$} \\
\hline No & 189 & $(62.8)$ & 112 & $(45.5)$ & 23.1 & $<0.001$ \\
\hline Sometimes & 105 & $(34.9)$ & 111 & $(45.1)$ & & \\
\hline Always & 7 & (2.3) & 23 & $(9.4)$ & & \\
\hline \multicolumn{7}{|l|}{ Empowerment } \\
\hline \multicolumn{7}{|l|}{ Medication $(n=548)$} \\
\hline Without prescription & 19 & (6.3) & 4 & $(1.6)$ & 7.3 & 0.007 \\
\hline With prescription & 283 & $(93.7)$ & 242 & $(98.4)$ & & \\
\hline \multicolumn{7}{|c|}{ Self-management during illness $(n=548)$} \\
\hline Yes & 67 & $(22.2)$ & 80 & $(32.5)$ & 7.4 & 0.007 \\
\hline No & 235 & $(77.8)$ & 166 & $(67.5)$ & & \\
\hline \multicolumn{7}{|l|}{ Seeking a doctor $(n=547)$} \\
\hline Yes & 228 & $(75.5)$ & 167 & $(68.2)$ & 3.6 & 0.057 \\
\hline No & 74 & $(24.5)$ & 78 & $(31.8)$ & & \\
\hline
\end{tabular}


Table 3 Estimations and statistics of selected predictors as revealed by multiple logistic regression

\begin{tabular}{|c|c|c|c|c|c|}
\hline Determining factors & $\boldsymbol{\beta}$ & SE & Adjusted ORs (95\% Cls) & $P$ value & HL point \\
\hline \multicolumn{6}{|l|}{ Socioenvironmental determinants } \\
\hline \multicolumn{6}{|l|}{ Dominant spoken dialect } \\
\hline Taiwanese, Hakka or other dialect & 0.76 & 0.11 & 2.13 (1.72 to 2.64$)$ & $<0.001$ & +1 \\
\hline Mandarin & Ref. & & & & \\
\hline \multicolumn{6}{|l|}{ Health service use } \\
\hline \multicolumn{6}{|l|}{ Having family doctors } \\
\hline No & 0.38 & 0.19 & $1.46(1.00$ to 2.14$)$ & 0.049 & +1 \\
\hline Yes & Ref. & & 1.00 & & \\
\hline \multicolumn{6}{|l|}{ Health costs } \\
\hline \multicolumn{6}{|l|}{ Self-paid pneumonia vaccination } \\
\hline No & 0.25 & 0.10 & $1.28(1.05$ to 1.57$)$ & 0.016 & +1 \\
\hline Yes & Ref. & & 1.00 & & \\
\hline \multicolumn{6}{|l|}{ Health behaviours } \\
\hline \multicolumn{6}{|l|}{ Searching online health information } \\
\hline No & 0.21 & 0.10 & 1.24 (1.01 to 1.52$)$ & 0.039 & +1 \\
\hline Yes & Ref. & & 1.00 & & \\
\hline \multicolumn{6}{|l|}{ Health outcomes } \\
\hline \multicolumn{6}{|l|}{ Assistance while visiting a doctor } \\
\hline Need assistance & 0.53 & 0.19 & 1.70 (1.16 to 2.48$)$ & 0.006 & +1 \\
\hline No assistance needed & Ref. & & 1.00 & & \\
\hline \multicolumn{6}{|l|}{ Activities of daily living } \\
\hline Having difficulty & 0.46 & 0.24 & 1.58 (1.00 to 2.52$)$ & 0.052 & +1 \\
\hline No difficulty & Ref. & & 1.00 & & \\
\hline \multicolumn{6}{|l|}{ Participation } \\
\hline \multicolumn{6}{|l|}{ Attending health classes } \\
\hline No & 0.32 & 0.11 & 1.38 (1.12 to 1.70$)$ & 0.003 & +1 \\
\hline Yes & Ref. & & 1.00 & & \\
\hline \multicolumn{6}{|l|}{ Empowerment } \\
\hline \multicolumn{6}{|l|}{ Self-management during illness } \\
\hline No & 0.25 & 0.10 & 1.28 (1.05 to 1.57$)$ & 0.016 & +1 \\
\hline Yes & Ref. & & 1.00 & & \\
\hline
\end{tabular}

The goodness of fit is measured by McFadden's $R^{2}=0.27$ and $p$ value of 0.92 in the Hosmer-Lemeshow test $(n=552,85 \%)$. $\mathrm{HL}$, health literacy.

HL) associated with the HL level $(p<0.1)$ were identified using Pearson's $\chi^{2}$ test (table 2).

These 24 factors including personal determinants, situational determinants, socioenvironmental determinants, health service use, health costs, health behaviours, health outcomes, participation and empowerment were entered in multiple logistic regression, as shown in table 2. Limited HL was significantly associated with less health service use or self-paid vaccination in preventive medicine, such as not having a family doctor (adjusted OR (AOR) 1.46, 95\% CI 1.00 to 2.14) or not receiving self-paid vaccination (AOR $1.28,95 \%$ CI 1.05 to 1.57 ). In addition, older adults with poorer health behaviour regarding the items related to searching online health information (AOR 1.24, 95\% CI 1.01 to 1.52 ), less social participation while attending health classes (AOR 1.38, 95\% CI 1.12 to 1.70 ) and poor empowerment of selfmanagement during illness (AOR 1.28, 95\% CI 1.05 to 1.57) had limited HL. Moreover, poorer health outcomes in older adults, such as having difficulty in performing daily living activities (AOR 1.58, 95\% CI 1.00 to 2.52) and requiring assistance while seeing a doctor (AOR 1.70, 95\% CI 1.16 to 2.48), may be associated with limited HL. In particular, older adults whose dominant dialect was a dialect other than Mandarin had a higher odds of having limited HL (AOR 2.13, 95\% CI 1.72 to 2.64; table 3). The indicators of model performance revealed a reasonably good fit in the training data set, including an acceptable pseudo $R^{2}$ value of 0.27 and nonsignificance $(p=0.923)$ in the Hosmer-Lemeshow test. 
Table 4 Overall accuracy of limited health literacy classification with various cut-off points (optimal cut-off $=5$ )

\begin{tabular}{|c|c|c|c|c|c|}
\hline \multirow{2}{*}{$\begin{array}{l}\text { Cut-off } \\
\text { value }\end{array}$} & \multicolumn{5}{|l|}{ Test data set $(n=96,15 \%)^{*}$} \\
\hline & Overall accuracy† n (\%) & Sensitivity \% & Specificity \% & Positive predictive value $\%$ & Negative predictive value $\%$ \\
\hline 1 & $50(54.3)$ & 100.0 & 0.0 & 54.3 & NA $\ddagger$ \\
\hline 2 & $51(55.4)$ & 100.0 & 2.4 & 54.9 & 100.0 \\
\hline 3 & $53(57.6)$ & 90.0 & 19.0 & 57.0 & 61.5 \\
\hline 4 & $57(62.0)$ & 74.0 & 47.6 & 62.7 & 60.6 \\
\hline 5 & $63(68.5)$ & 62.0 & 76.2 & 75.6 & 62.7 \\
\hline 6 & $58(63.0)$ & 38.0 & 92.9 & 86.4 & 55.7 \\
\hline 7 & $44(47.8)$ & 4.0 & 100.0 & 100.0 & 46.7 \\
\hline 8 & $43(46.7)$ & 2.0 & 100.0 & 100.0 & 46.2 \\
\hline
\end{tabular}

*Only 92 participants finished all responses in the measurement to present the classification accuracy. †Agreement between predicted and observed level health literacy (limited or sufficient). ‡NA: not available; denominator is zero.

NA, not available.

Eight variables were selected using the forward stepwise selection method in the multivariate model and were used to calculate the HL score. Cross-validation was performed by including $15 \%$ participants $(n=96 / 648)$, of whom 92 provided all responses for the measurement of prediction accuracy. The overall accuracy in predicting limited HL with various cut-off points is listed in table 4 . The optimal cut-off point was considered to be 5 , yielding sensitivity and specificity of $62.0 \%$ and $76.2 \%$, respectively. By using a score of 5 of 8 to predict the limited HL level, the obtained PPV and NPV were $75.6 \%$ and $62.7 \%$, respectively. Figure 2 presents the predictive ability of the scoring algorithm among older adults in the test data set. Figure 3 shows the indicators of model performance revealed reasonably satisfactory performance with an AUC of 0.71 (95\% CI 0.61 to 0.81 ).

\section{DISCUSSION AND CONCLUSION \\ Discussion}

To the best of our knowledge, this is the first study to develop a model for predicting the HL level of communitydwelling older adults. This algorithm-based model was well calibrated by integrating HL-related factors from the model of the HLS-EU Consortium and was useful for HL risk prediction among older adults. In addition, this model exhibited a moderate ability for discriminating between older adults with sufficient HL and limited HL.

In this study, we integrated variables associated with both medical and public health perspectives from the aforementioned HL model of the HLS-EU Consortium and proposed a simple scoring algorithm. The scoring system dichotomises older adults into high-risk (cutoff $\geq 5$ ) and low-risk (cutoff $<5$ ) populations to maximise the sensitivity and specificity of the prediction of limited HL. Therefore, early identification using five or more factors from a total of eight significant HL predictors among older adults can be an effective strategy that can be implemented in future clinical practice. On the basis of proposed cut-off points, among the 92 older adults included in the test data set, $63(68.5 \%)$ with a cut-off value of $\geq 5$ were recommended to undergo further HL intervention, although only 31 $(62.0 \%)$ actually had limited HL, resulting in a PPV of $75.6 \%$. Given the importance of early identification and strategy provision for community-dwelling older adults

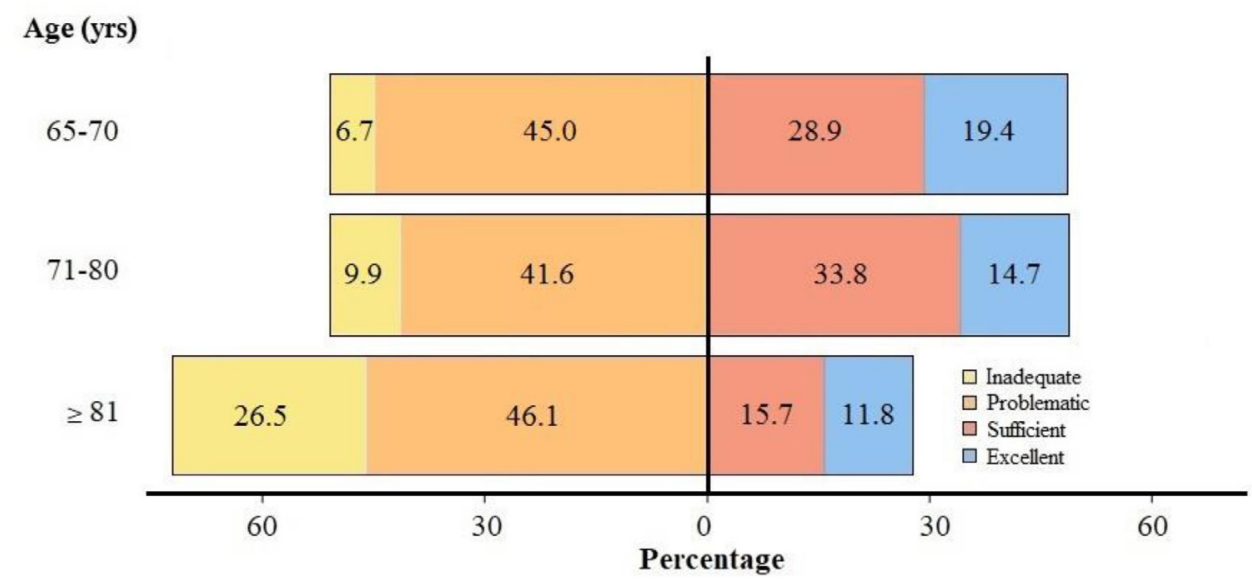

Figure 2 Age-specific health literacy levels. 


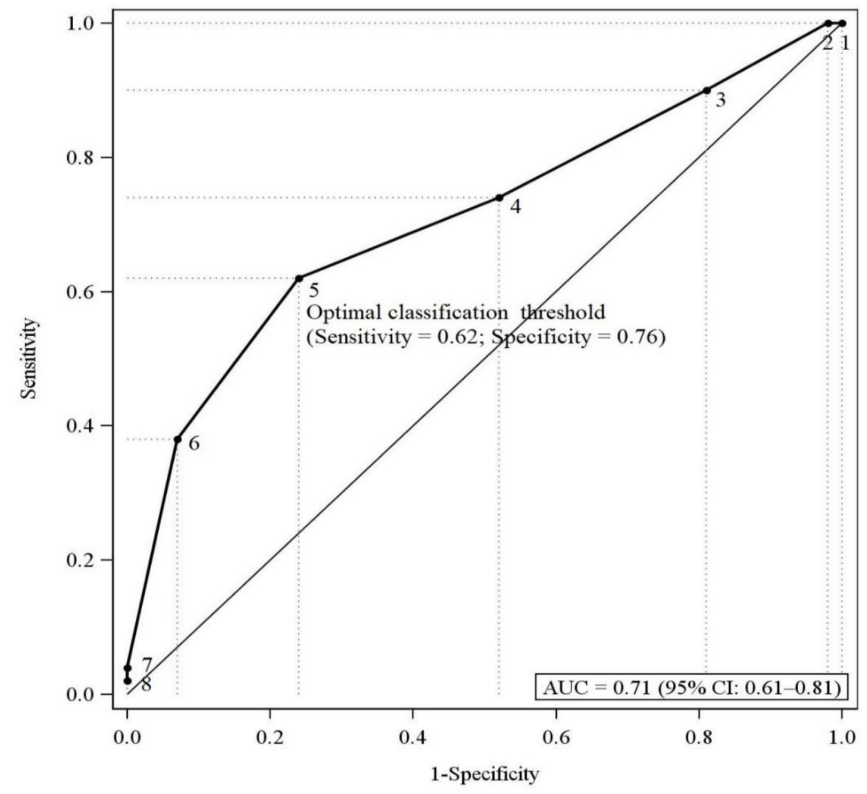

Figure 3 ROC curve and c-statistics of the fitting test in the test data set. The AUC was 0.71 (95\% Cl 0.61 to 0.81), indicating acceptable discrimination. AUC, area under the curve; ROC, receiver operating characteristic.

with a high risk of limited HL, the proposed scoring algorithm can be considered useful in community practice.

A previous study proposed using $80 \%-90 \%$ of data to build a prediction model and $10 \%-20 \%$ of data to validate the model. ${ }^{38}$ In our study, we followed two previous studies and accordingly divided our data into a training data set (by using $85 \%$ of the data) and a test data set (by using $15 \%$ of the data). ${ }^{39} 40$ In addition, we randomly selected $10 \%(n=64)$ and $20 \%(n=128)$ of the data to examine the reliability of dividing our data into $85 \%$ and $15 \%$ in our study. Similar validation results were obtained when we included $10 \%(\mathrm{n}=64), 15 \%(\mathrm{n}=96)$, and $20 \%$ $(\mathrm{n}=128)$ of the participants. The overall accuracy was $75.0 \%(48 / 64), 68.5 \%(63 / 96)$ and $64.8 \%(83 / 128)$ for $10 \%, 15 \%$, and $20 \%$ of the participants, respectively, indicating that the score of 5 was the optimal cut-off point.

This conceptual framework integrating medical and public health perspectives developed by the HLS-EU Consortium is suitable for determining the most relevant determinants of the HL level in older adults. Eight predictors were identified to be significantly associated with the HL level: one socioenvironmental determinant (ie, dominant spoken dialect) and seven HL-related factors including health services (ie, having a family doctor), health cost (ie, self-paid pneumonia vaccination), health behaviours (ie, searching online health information), health outcomes (ie, assistance while visiting a doctor and performing activities of daily living), participation (ie, attending health classes) and empowerment (ie, selfmanagement during illness). The results for the seven identified predictors of HL-related factors are consistent with those of previous studies, for example, having a family doctor, ${ }^{8}$ costs for self-paid vaccination, ${ }^{41}$ searching online health information, ${ }^{10}$ functional status such as difficulty in performing daily activities and assistance while visiting doctors, ${ }^{41}{ }^{42}$ participation in health classes ${ }^{43}$ and self-efficacy in disease management. ${ }^{44}$ However, our study result revealed that personal and situational factors did not affect HL among older adults. Previous studies have reported that personal determinants, namely age, educational level and working status, as well as situational and environmental determinants, including marriage and residential area, were significantly associated with the HL level. ${ }^{15} 192045$ This difference might be because personal and situational determinants were the proximal factors of HL that are affected and displaced by a more distal and upstream factor (societal and environmental determinants). ${ }^{46}$

Some studies have developed a weighted score based on multiple regression to determine the HL level. These studies have used originally estimated beta values or have transformed the beta values into a score of multivariate regression. ${ }^{19} 2047$ For example, Miller et al used 20 variables to establish a nonequal weighted HL scoring system for older adults, and their scoring system correctly classified the HL of $73.2 \%$ of the participants. ${ }^{20}$ Our simple scoring algorithm referred to associations (positive and negative) between the HL level and risk factors with equal weighs $(+1$ or -1$)$ to identify individuals with limited HL. ${ }^{31}$ Our algorithm is rapid and straightforward to use and can be widely implemented for determining the HL of older adults in clinical practice. Our results from the eight questions (correctly classified the HL of $65.8 \%$ of the participants) can help rapidly determine the target intervention tailored for a specific older adult.

Users can rapidly predict limited HL through an evaluation of their HL-related personal, situational and environmental factors as well as health behaviour and outcomes by using our algorithm and thus identify communitydwelling older adults who may require further health assistance. Hospitalisation and mortality due to poor HL in older adults can be prevented through early identification and intervention. Therefore, this assessment tool should be promptly extended to broader communities.

Our study has some limitations. First, in this crosssectional study, participants were recruited from northern, central and southern Taiwan by using the convenience sampling method. Therefore, potential selection bias might exist. In addition, although we included comprehensive HL-related factors on the basis of a conceptual model of HL, some crucial situational or socioenvironmental factors related to the situational demand or organisational environment might be difficult to measure because information on the organisational environment was not available in our individual interview study. Second, this study relied on the 47-item HLSEU-Q self-reported questionnaire for determining HL. Additional objective HL assessments might be required to recognise functional HL for preventing the potential for outcome misclassification bias. Third, the high prevalence rate of limited HL (54.9\%) in our sample may 
affect the prediction ability (ie, PPV) of this algorithm when applied in other populations. Therefore, when this algorithm is applied to a population with a lower prevalence of limited HL, older adults with positive results of limited HL may in fact have sufficient HL. Furthermore, we excluded older adults who could not pass the Mini-Cog screening or follow instructions to complete the assessment. Therefore, our model may not be generalisable to the entire population of older adults. Thus, this model is not recommended to be used in individuals with cognitive impairment or dementia, who may have difficulty understanding instructions. Larger population studies with prospective longer-term outcome measures are necessary to validate our study findings.

\section{Conclusion}

We proposed a simple clinical scoring algorithm with substantial sensitivity and satisfactory specificity to assess the risk of limited HL among community-dwelling older adults.

\section{Practice implications}

This scoring algorithm can not only help clinicians to assess and identify the HL level among older adults but also assist researchers to establish intervention strategies for older adults with limited HL. However, for further population-based application for the early detection of older adults with a high risk of limited HL, prospective trials should examine the implementation and utility of this algorithm in the community.

\section{Author affiliations}

${ }^{1}$ Master's Program in Long-Term Care \& School of Gerontology Health Management, College of Nursing, Taipei Medical University, Taipei, Taiwan

${ }^{2}$ Department of Geriatric Medicine \& Physical Medicine and Rehabilitation, Taipei Medical University Hospital, Taipei, Taiwan

${ }^{3}$ Cochrane Taiwan, Taipei Medical University, Taipei, Taiwan

${ }^{4}$ Graduate Institute of Clinical Medicine, College of Medicine, Taipei Medical

University, Taipei, Taiwan

${ }^{5}$ Department of Orthopedic Surgery, National Taiwan University Hospital, Taipei, Taiwan

${ }^{6}$ National Institute of Environmental Health Sciences, National Health Research Institutes, Miaoli, Taiwan

${ }^{7}$ Health Policy and Care Research Center, College of Public Health, Taipei Medical University, Taipei, Taiwan

${ }^{8}$ School of Health Care Administration, Taipei Medical University, Taipei, Taiwan

${ }^{9}$ Department of Geriatrics and Gerontology, National Taiwan University Hospital, Taipei, Taiwan

${ }^{10}$ Superintendent Office, National Taiwan University Hospital Chutung Branch, Hsinchu, Taiwan

${ }^{11}$ Department of Family Medicine, Taipei Medical University Hospital, Taipei, Taiwan

${ }^{12}$ School of Public Health,College of Public Health, Taipei Medical University, Taipei, Taiwan

${ }^{13}$ Department of Family Medicine, School of Medicine, College of Medicine, Taipei Medical University, Taipei, Taiwan

${ }^{14}$ Department of Physical Medicine and Rehabilitation, National Taiwan University Hospital Beihu Branch, Taipei, Taiwan

${ }^{15}$ Health Science and Wellness Center, National Taiwan University, Taipei, Taiwan

${ }^{16}$ Department of Intensive Care Medicine, Chi Mei Medical Center, Tainan, Taiwan

${ }^{17}$ Department of Public Health, National Cheng Kung University College of Medicine, Tainan, Taiwan

${ }^{18}$ Faculty of Public Health, Department of Epidemiology, Universitas Airlangga, Surabaya, Indonesia
${ }^{19}$ Department of Public Health, College of Public Health, China Medical University, Taichung, Taiwan

Acknowledgements We thank Wu-Shou Chang from the Asian Health Literacy Association for authorising the use of the Mandarin Chinese version of the HLS-EU-Q47.

Contributors W-HH, Y-MC and KNK designed the study. C-TS, D-SH and D-CC acquired of data. H-NS, M-JC and H-WT analysed the data. W-HH, H-NS, and H-WT drafted the manuscript. C-TS, D-SH, D-CC, KNK and C-YL reviewed and revised the manuscript. The author (s) read and approved the final manuscript. C-YL is the overall guarantor.

Funding This research was supported by research grants from the Taiwan National Health Research Institutes (MOHW107-TDU-M-212-133001-107-FR-04), Chi Mei Medical Center (108CM-TMU-01), and Taipei Meidcal University Hospital (109TMUH-H-01).

Disclaimer The funder was not involved in study design, and will not be involved in the collection, analysis or interpretation of data.

Competing interests None declared.

Patient consent for publication Not applicable.

Ethics approval This study was approved by Taipei Medical University-Joint Institutional Review Board (N201804046) and National Taiwan University Hospital (201804057RIND).

Provenance and peer review Not commissioned; externally peer reviewed.

Data availability statement Data are available upon reasonable request. All data relevant to the study are included in the article or uploaded as online supplemental information.

Supplemental material This content has been supplied by the author(s). It has not been vetted by BMJ Publishing Group Limited (BMJ) and may not have been peer-reviewed. Any opinions or recommendations discussed are solely those of the author(s) and are not endorsed by BMJ. BMJ disclaims all liability and responsibility arising from any reliance placed on the content. Where the content includes any translated material, BMJ does not warrant the accuracy and reliability of the translations (including but not limited to local regulations, clinical guidelines, terminology, drug names and drug dosages), and is not responsible for any error and/or omissions arising from translation and adaptation or otherwise.

Open access This is an open access article distributed in accordance with the Creative Commons Attribution Non Commercial (CC BY-NC 4.0) license, which permits others to distribute, remix, adapt, build upon this work non-commercially, and license their derivative works on different terms, provided the original work is properly cited, appropriate credit is given, any changes made indicated, and the use is non-commercial. See: http://creativecommons.org/licenses/by-nc/4.0/.

\section{ORCID iDs}

Wen-Hsuan Hou http://orcid.org/0000-0002-4376-6298

Chung-Yi Li http://orcid.org/0000-0002-0321-8908

\section{REFERENCES}

1 Sørensen K, Van den Broucke S, Fullam J, et al. Health literacy and public health: a systematic review and integration of definitions and models. BMC Public Health 2012;12:80.

2 Weiss BD, Mays MZ, Martz W, et al. Quick assessment of literacy in primary care: the newest vital sign. Ann Fam Med 2005;3-514-22. doi:10.1370/afm.405

3 Heinrich C. Health literacy: the sixth vital sign. J Am Acad Nurse Pract 2012;24:223 doi:10.1111/j.1745-7599.2012.00698.x

4 Lee S-YD, Tsai T-I, Tsai Y-W, et al. Health literacy, health status, and healthcare utilization of Taiwanese adults: results from a national survey. BMC Public Health 2010;10:614. doi:10.1186/1471-2458-10614

5 Shen H-N, Lin C-C, Hoffmann T, et al. The relationship between health literacy and perceived shared decision making in patients with breast cancer. Patient Educ Couns 2019;102:360-6. doi:10.1016/j. pec.2018.09.017

6 Prevention CfDCa. Improving health literacy for older adults: expert panel report 2009, 2009.

7 Sørensen K, Pelikan JM, Röthlin F, et al. Health literacy in Europe: comparative results of the European health literacy survey (HLS-EU). Eur J Public Health 2015;25:1053-8. doi:10.1093/eurpub/ckv043 
8 Duong VT, Lin I-F, Sorensen K, et al. Health literacy in Taiwan: a population-based study. Asia Pac J Public Health 2015;27:871-80. doi:10.1177/1010539515607962

9 Wolf MS, Gazmararian JA, Baker DW. Health literacy and functional health status among older adults. Arch Intern Med 2005;165:1946. doi:10.1001/archinte.165.17.1946

10 Liu Y-B, Liu L, Li Y-F. Relationship between health literacy, healthrelated behaviors and health status: a survey of elderly Chinese. Int $J$ Environ Res Public Health 2015:12:9714-25.

11 Hou WH, Huang YJ, Lee Y. Validation of the integrated model of health literacy in patients with breast cancer. Cancer Nurs 2017.

12 Nutbeam D. Health literacy as a public health goal: a challenge for contemporary health education and communication strategies into the 21st century. Health Promot Int 2000;15:259-67.

13 Lee S-YD, Tsai T-I, Tsai Y-W, et al. Health literacy and women's health-related behaviors in Taiwan. Health Educ Behav 2012;39:210-8. doi:10.1177/1090198111413126

14 Palumbo R. Examining the impacts of health literacy on healthcare costs. An evidence synthesis. Health Serv Manage Res 2017;30:197-212. doi:10.1177/0951484817733366

15 Chung $\mathrm{M}-\mathrm{H}$, Chen L-K, Peng L-N, et al. Development and validation of the health literacy assessment tool for older people in Taiwan: potential impacts of cultural differences. Arch Gerontol Geriatr 2015;61:289-95. doi:10.1016/j.archger.2015.06.015

16 O'Connor PJ, Martin B, Weeks CS, et al. Factors that influence young people's mental health help-seeking behaviour: a study based on the health belief model. J Adv Nurs 2014;70:2577-87. doi:10.1111/jan.12423

17 Zarcadoolas C, Pleasant A, Greer DS. Understanding health literacy: an expanded model. Health Promot Int 2005;20:195-203. doi:10.1093/heapro/dah609

18 Altin SV, Finke I, Kautz-Freimuth S, et al. The evolution of health literacy assessment tools: a systematic review. BMC Public Health 2014:14:1207. doi:10.1186/1471-2458-14-1207

19 Hanchate AD, Ash AS, Gazmararian JA, et al. The demographic assessment for health literacy (Dahl): a new tool for estimating associations between health literacy and outcomes in national surveys. J Gen Intern Med 2008;23:1561-6. doi:10.1007/s11606008-0699-5

20 Miller MJ, Degenholtz HB, Gazmararian JA, et al. Identifying elderly at greatest risk of inadequate health literacy: a predictive model for population-health decision makers. Res Social Adm Pharm 2007;3:70-85. doi:10.1016/j.sapharm.2006.06.001

21 Borson S, Scanlan JM, Chen P, et al. The Mini-Cog as a screen for dementia: validation in a population-based sample. J Am Geriatr Soc 2003;51:1451-4. doi:10.1046/j.1532-5415.2003.51465.x

22 Everitt BS. Multivariate analysis: the need for data, and other problems. Br J Psychiatry 1975;126:237-40. doi:10.1192/ bjp.126.3.237

23 Shih YT, Hung YT, Chang HY. The design, contents, operation and the characteristics of the respondents of the 2001 National health interview survey in Taiwan. Taiwan J Public Health 2003;22:419-30.

24 van der Heide I, Rademakers J, Schipper M, et al. Health literacy of Dutch adults: a cross sectional survey. BMC Public Health 2013;13:179. doi:10.1186/1471-2458-13-179

25 Toçi E, Burazeri G, Sørensen K, et al. Concurrent validation of two key health literacy instruments in a South Eastern European population. Eur J Public Health 2015;25:482-6. doi:10.1093/eurpub/ cku190

26 Nakayama K, Osaka W, Togari T, et al. Comprehensive health literacy in Japan is lower than in Europe: a validated Japanese-language assessment of health literacy. BMC Public Health 2015;15:505. doi:10.1186/s12889-015-1835-x

27 Acharya S, Pant AK, Gyawali PK. Deep learning based large scale Handwritten Devanagari character recognition. 2015 9th International
Conference on software, knowledge, information management and applications (Skima) 2015.

28 Zhang Z. Model building strategy for logistic regression: purposeful selection. Ann Transl Med 2016;4:111. doi:10.21037/atm.2016.02.15

29 Wollert KC, Kempf T, Lagerqvist B, et al. Growth differentiation factor 15 for risk stratification and selection of an invasive treatment strategy in non ST-elevation acute coronary syndrome. Circulation 2007;116:1540-8. doi:10.1161/CIRCULATIONAHA.107.697714

30 Calvo C, García-García ML, Blanco C, et al. Multiple simultaneous viral infections in infants with acute respiratory tract infections in Spain. J Clin Virol 2008;42:268-72.

31 Chen P-L, Lee C-C, Li C-Y, et al. A simple scoring algorithm predicting vascular infections in adults with nontyphoid Salmonella bacteremia. Clin Infect Dis 2012;55:194-200. doi:10.1093/cid/cis381

32 Wicki J, Perneger TV, Junod AF, et al. Assessing clinical probability of pulmonary embolism in the emergency ward: a simple score. Arch Intern Med 2001;161:92-7. doi:10.1001/archinte.161.1.92

33 Menon P, Geevasinga N, Yiannikas C, et al. Sensitivity and specificity of threshold tracking transcranial magnetic stimulation for diagnosis of amyotrophic lateral sclerosis: a prospective study. Lancet Neurol 2015;14:478-84. doi:10.1016/S1474-4422(15)000149

34 Hanley JA, McNeil BJ. The meaning and use of the area under a receiver operating characteristic $(\mathrm{ROC})$ curve. Radiology 1982;143:29-36. doi:10.1148/radiology.143.1.7063747

35 Menard S. Coefficients of determination for multiple logistic regression analysis. Am Stat 2000;54.

36 Nalls MA, McLean CY, Rick J, et al. Diagnosis of Parkinson's disease on the basis of clinical and genetic classification: a population-based modelling study. Lancet Neurol 2015;14:1002-9. doi:10.1016/S14744422(15)00178-7

37 Aggarwal R, Ranganathan P. Understanding diagnostic tests - part 3: receiver operating characteristic curves. Perspect Clin Res 2018;9:145-8. doi:10.4103/picr.PICR_87_18

38 Tropsha A. Best practices for QSAR model development, validation, and exploitation. Mol Inform 2010;29:476-88. doi:10.1002/ minf.201000061

39 Deep Learning Based Large Scale Handwritten Devanagari Character Recognition. 2015 9th International Conference on software, knowledge, information management and applications (SKIMA); 2015 15-17 Dec 2015

40 Kusiak A, Tang F, Xu G. Multi-objective optimization of HVAC system with an evolutionary computation algorithm. Energy 2011;36:2440-9. doi:10.1016/j.energy.2011.01.030

41 Vogt D, Schaeffer D, Messer M, et al. Health literacy in old age: results of a German cross-sectional study. Health Promot Int 2018;33:739-47. doi:10.1093/heapro/dax012

42 Paasche-Orlow MK, Wolf MS. The causal pathways linking health literacy to health outcomes. Am J Health Behav 2007;31:19-26.

43 Manafo E, Wong S. Health literacy programs for older adults: a systematic literature review. Health Educ Res 2012;27:947-60. doi:10.1093/her/cys067

44 Chen J-Z, Hsu H-C, Tung H-J, et al. Effects of health literacy to selfefficacy and preventive care utilization among older adults. Geriatr Gerontol Int 2013;13:70-6. doi:10.1111/j.1447-0594.2012.00862.x

45 Huang Y-J, Wang Y-L, Wu T-Y, et al. Validation of the short-form health literacy scale in patients with stroke. Patient Educ Couns 2015;98:762-70. doi:10.1016/j.pec.2015.02.021

46 Krieger N. Proximal, distal, and the politics of causation: what's level got to do with it? Am J Public Health 2008;98:221-30. doi:10.2105/ AJPH.2007.111278

47 Wicki J, Perneger TV, Junod AF, et al. Assessing clinical probability of pulmonary embolism in the emergency ward: a simple score. Arch Intern Med 2001;161:92. doi:10.1001/archinte.161.1.92 